\title{
The Complex Variability of Climate
}

\author{
Climate scientist Michael Ghil describes gaps in our understanding of \\ climate change.
}

By Erika K. Carlson

7 he world is heating up. But it's not just average temperature that matters. To fully understand and model climate change, it's important to also understand the climate's variability, says climate physicist Michael Ghil, who has positions at the University of California, Los Angeles, and at the École Normale Supérieure in Paris. Climate variability-the short-term ups and downs of atmospheric conditions that include extreme events like hurricanes-is hard to separate from the long-term trends of climate change. But doing so is important if physicists are to contribute to teasing out the impacts of human activity on the climate, something that Ghil hopes his studies on climate variability and climate change will do.

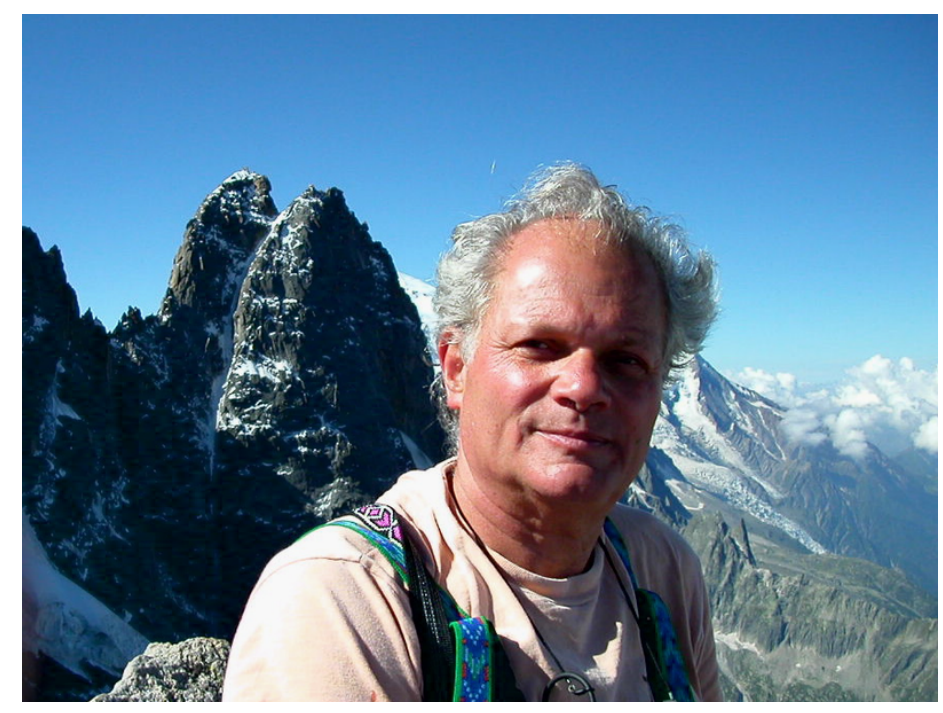

Credit: Courtesy of M. Ghil
Ghil and his colleague Valerio Lucarini of the University of Hamburg in Germany and the University of Reading in the UK today published the first review on climate physics for Reviews of Modern Physics. In a conversation with Physics, Ghil explains gaps in our understanding of climate change and describes how he hopes his new paper will contribute to the field.

\section{All interviews are edited for brevity and clarity.}

\section{What made you decide to write this review paper?}

A huge challenge the research community faces is dealing with uncertainties in future climate projections. Addressing these uncertainties, particularly in the climate's response to human activity, is a really important issue. And that's one of the key things that we address in this paper.

There are two basic methodologies, or areas of science, whose use we discuss. From the math side, dynamical systems theory, and on the physics side, statistical mechanics (specifically fluctuation-dissipation and linear response). There are many papers that individually discuss the use of these methods in studying climate, but not one that looks at them both. This paper, written by Valerio and me, attempts to do that.

\section{What message do you hope your readers will take away from this paper?}

Unfortunately, there is a perception-perpetuated by some climate science activists - that, "the science is done when it comes to climate change; now society needs to act." I agree that we do need to act; there's plenty of evidence for that. But the science is very far from done. We're only just getting to the really hard, fundamental questions. 


\section{Can you elaborate?}

Scientists have a good understanding of how human activities are modifying the mean global temperature. But we are missing a full understanding of how they impact other aspects of climate-for example, the amplitude and frequency of extreme events.

One of the issues is that most people, scientists or otherwise, think of climate as something static that anthropogenic factors are just pushing around. But the natural climate constantly fluctuates. What I hope Valerio and I offer in this paper is a unified framework for modeling climate change and climate variability that accounts for these fluctuations.

\section{What is the difference between weather and climate?}

Weather can be defined as the condition of the atmosphere on a timescale shorter than a season, or about 100 days. The state of the atmosphere and ocean on a timescale beyond ten years is certainly climate. Somewhere in between, the two overlap. Unfortunately, there's no easy way to distinguish exactly where weather stops and climate starts.

Think about the seasons, whose onward march is the most dramatic natural climate phenomenon. There can be a bigger difference in the weather between summer and winter in places such as New York, for example, where the temperature drops below freezing in winter and rises above $30^{\circ} \mathrm{C}$ in summer, than there is between the climate today and a century ago. But the march of the seasons is changing from one decade to the next. And how you enter a winter, for example with a warmer or a colder ocean, may affect the number or intensity of particularly cold episodes.

\section{Can you say more about how climate on long timescales is related to the seasons or to extreme events?}

Global warming is accompanied by a reduction in the equator-to-pole temperature difference, since the temperature is rising more in the polar regions than in the equatorial ones. This reduction can increase the number of extreme events, such as the recent polar-vortex-induced cold snaps in the Midwest and Eastern US. But this conjecture is still hotly debated.

The climate system is complex, and there are unsolved problems in understanding how it behaves that merit attention. The fact is that human activity is affecting more than the mean temperature; we're affecting the march of the seasons and the amplitude, duration, and frequency of extreme weather events.

\section{What was the main challenge in putting together a review paper on such an interdisciplinary field?}

Keeping it to only 77 pages and 52 figures.

Erika K. Carlson is a Corresponding Editor for Physics based in New York City. 